\title{
The Sense of Object-Presence with Projection-Augmented Models
}

\author{
Brett Stevens and Dr Jennifer Jerrams-Smith \\ Department of Information Systems, University of Portsmouth \\ Burnaby Terrace, 1-8 Burnaby Road \\ Portsmouth, Hampshire, UK. PO1 3AE \\ +4402392846435 \\ brett.stevens@port.ac.uk_jenny.jerrams-smith@port.ac.uk \\ http://www.tech.port.ac.uk/ stevensb/
}

\begin{abstract}
Projection-augmented models are a type of non-immersive, coincident haptic and visual display that uses a physical model as a three dimensional screen for projected visual information. Supporting two sensory modalities consistently should create a strong sense of the object's existence. However, conventional measures of presence have only been defined for displays that surround and isolate a user from the real world.

The idea of object-presence is suggested to measure 'the subjective experience that a particular object exists in a user's environment, even when that object does not'. This definition is more appropriate for assessing non-immersive displays such as projection-augmented models.
\end{abstract}

\section{Keywords}

Presence, object-presence, projection-augmented models, coincident haptic and visual display, non-immersive display

\section{INTRODUCTION}

Virtual reality was originally conceived as an advanced human-computer interface that immersed a user within a realistic three dimensional environment [12]. However, this form of immersive virtual reality requires expensive equipment and can have negative side effects for a user [2]. Therefore, it has been suggested that virtual reality should be redefined as any "advanced human-computer interface that simulates a realistic environment and allows participants to interact with it." [8].

This definition includes non-immersive displays such as conventional computer monitors as well as projectionaugmented models. This prototype display uses a physical model to act as a three dimensional screen for projected visual information.

One of immersive virtual reality's key benefits is its ability to induce a sense of presence, defined by Witmer \& Singer [14] as the "subjective experience of being in one place or environment, even when one is physically situated in another". However, this paper suggests an alternative definition for presence that is more appropriate for nonimmersive displays.

\section{OBJECT-PRESENCE}

Witmer \& Singer [14] state that presence in a virtual environment, is dependent on immersion and involvement. Whilst Slater \& Wilbur [11] suggest that one of the key components of immersion is the extent to which a virtual environment surrounds the user. However, a virtual environment is constructed from objects, which permits the definition of presence to be re-written as "the subjective experience of being co-located with a set of objects, even when one is physically not in such a situation". If this definition is used, the implication that the user should be surrounded, inherent in the concept of environment, is replaced with the idea that a user should have a feeling of being 'with' an object.

Considering the other components of immersion as suggested by Slater \& Wilbur [11]. The quality of a display (vivid), the range of sensory modalities (extensive) and the correspondence between the user's actions and displayed information (matching) are all aspects of how naturally a display supports a user. These components are not unique requirements for immersive displays. Indeed the only other factor unique to immersion apart from the ability to surround a user, is the extent to which a user is removed from reality (inclusion). Thus the difference in presence between immersive and non-immersive displays results from a display surrounding and isolating a user. However, some tasks do not require the user to be surrounded or isolated.

Presence forms an important subjective measure of a user's virtual experience, although it is only useful in relation to performance [3]. It is assumed that the more natural the display feels, the greater its usefulness [9]. This naturalness may better enable a user to utilize 'real-world' skills in a virtual environment although it may also help to transfer learning from the virtual environment back into the real- 
world [9]. The conventional definition of presence suggests that non-immersive displays are inadequate, even for tasks that do not require the user to be surrounded and isolated. Therefore, a new measure is needed to assess presence for non-immersive displays that will more closely consider task requirements and how naturally a display supports a user. "It is here" is the idea that a display medium brings an object or person to the user [9]. This idea has only been investigated for conventional television programmes, where it assesses the belief that the actual object being displayed exists within the television set. However, this concept can be extended to provide a measure for non-immersive displays where the object appears to be in the user's physical environment, instead of inside the display.
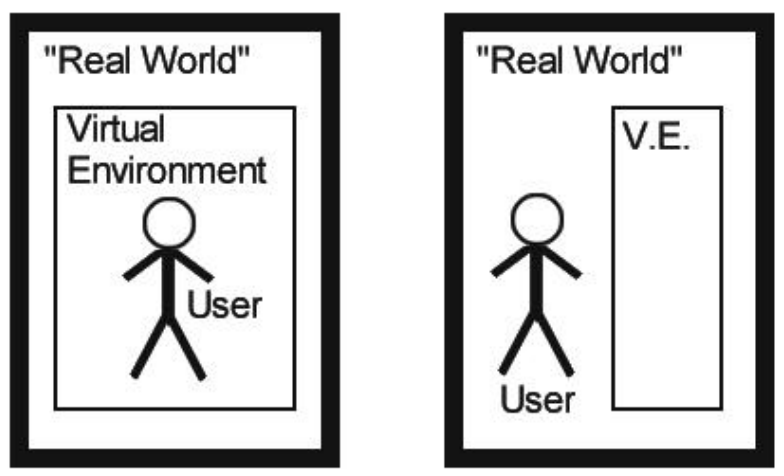

Figure 1 - Presence and Object-Presence.

Following the style used by Witmer \& Singer [10], "the subjective experience that a particular object exists in a user's environment, even when that object does not" will be termed 'Object-presence'. This definition does not distinguish between real or virtual environments although in the context of immersive virtual reality, object-presence and presence would be interdependent. More interestingly though, is the subjective experience that an object exists in the real-world. This can be thought of as a special case of virtual reality, where the user is co-located with a virtual environment (Figure 1). Presence and object-presence have a close relationship. Both have been conceptualised as types of transportation [9] where the user is either transported to the virtual environment or the virtual environment is transported to the user. This sense of object-presence is an important element for coincident haptic and visual displays that use a physical model as a three dimensional screen for projected visual information.

\section{PROJECTION-AUGMENTED MODELS}

Conventional virtual reality displays have the potential to present dynamic three dimensional objects, although they have a number of disadvantages. To support most physiological depth cues, and hence increase realism and scene depth, a user's "point of view" needs to be determined with some form of tracking device. This information is used to create and present an appropriate image for each eye although, because two images are presented, special glasses are needed to filter out the incorrect image [7]. To present the correct perspective information to each simultaneous user requires multiple sets of these devices, which can prove costly in terms of equipment, processing power and time [1]. Presenting haptic information is also a problem as the facilitating devices are generally low resolution or tend to either occlude the visual display or present the haptic information in a different spatial location [4]. An alternative solution would be to physically create the object under investigation, or for large objects a detailed scaled model. Although this would be expensive to create and difficult to modify, it would allow multiple simultaneous users to receive high resolution visual and haptic information from any perspective [10].

Projection-augmented models are a hybrid of these techniques where a simplified physical object acts as a three dimensional screen for a matching graphical model projected onto its surface [10]. The visual image can be altered easily like a conventional display but, because it is presented on the surface of a physical object, all physiological depth cues are supported for multiple users. The physical object can also be touched, which should provide coincident haptic and visual information, a sense that "what you see is what you feel". Supporting two sensory modalities consistently should create a strong sense of palpability, or awareness that the object exists [5], and hence a strong sense of object-presence. However, if the visual and haptic information is not consistent, for example if the visual information does not relate to the object's surface but to its inner workings or surrounding atmosphere, it will cause an intersensory discrepancy. This may result in either the visual, or haptic, information being ignored or in some cases, the incongruous information may be combined to create an inaccurate representation of the object [13].
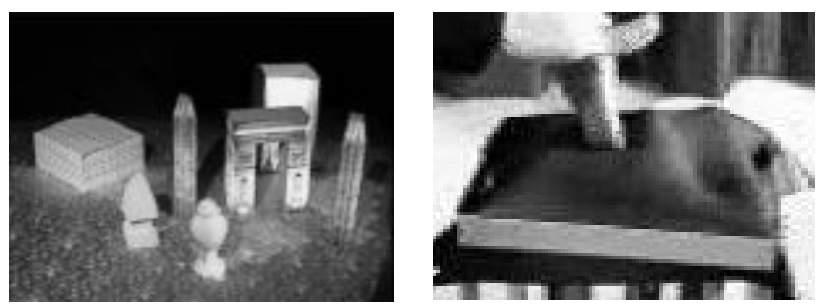

Figure 2 - "Table-top Spatially Augmented Reality" [10] and "The HapticScreen" [6].

The physical model could either be a static surrogate object [10] or a dynamically deforming physical simulation [6]. Both of these examples use relatively low resolution objects although the projected image provides a more realistic visual representation of the object's surface (Figure 2). In this context, object-presence is a measure of how much the presented object seems to exist, i.e. the combination of physical and visual information, not the existence of the physical object alone. 


\section{SUMMARY}

Projection augmented models offer a unique method for presenting visual and haptic information in the same spatial location. The visual information is projected onto a physical model which supports the ability to touch the object under investigation [10] and allows multiple simultaneous users to view it stereoscopically, without the need for head-tracking or stereoscopic glasses. Although only at the prototype stage, both static [10] and dynamic [6] models should allow a user to naturally access information.

One of the measures applied to a virtual reality display is the extent to which a user feels present. Linked to the idea of a display supporting the user in a "natural" way, it is assumed that the more natural the display feels the greater its usefulness [9]. This naturalness may enhance a user ability to utilize 'real-world' skills in a virtual environment although it may also help to transfer learning from the virtual environment back into the real-world. The conventional definition of presence requires a user to be isolated from the real-world and surrounded with a virtual environment. Although this definition is appropriate for some tasks, others do not require the creation of an entire environment.

Non-immersive displays can provide a realistic natural stimulus to a user even though they have a limited field of view. The idea of object-presence is suggested to measure the extent to which information presented with a nonimmersive display seems natural to a user. This concept replaces the feeling of being surrounded by an environment with the sense of being co-located with a collection of objects. This is more applicable to non-immersive displays and should provide an interesting measure for use with projection-augmented models.

Projection augmented models support nature interaction modes and should create a strong sense of object-presence. Future work includes the need to identify a measure of object-presence that is applicable to projection augmented models and to determine if a link between task performance and object-presence exists.

\section{ACKNOWLEDGMENTS}

We thank Miss Amanda Brightman, Dr David Callear, Dr Steve Hand and Dr David Heathcote for their comments and suggestions.

\section{REFERENCES}

1. Agrawala, M. et al., 1997. The two user responsive workbench: Support for collaboration through individual views of a shared space. In: Proceedings of SIGGRAPH '97: Computer Graphics Proceedings, Annual Conference Series. Los Angeles, California: USA. ACM Press, 327-332.
2. Cobb, S.V.G., Nichols, S., Ramsey, A., and Wilson, J.R., 1999. Virtual Reality-Induced Symptoms and Effects (VRISE). Presence Teleoperators and Virtual Environments, 8(2), 169-186.

3. Ellis, S.R., 1996. Presence of mind: A reaction to Thomas Sheridan's "Further musings on the psychophysics of presence". Presence Teleoperators and Virtual Environments, 5(2), 247-259.

4. Henderson, A., 1999. The nanoManipulator. [Online]. Available from http://www.cs.unc.edu/Research/nano/ [Accessed: 30 November 1999].

5. Hinckley, K., 1996. Haptic issues for virtual manipulation. Unpublished doctoral dissertation, University of Virginia.

6. Iwata, H., 1998. HapticScreen. In: Proceedings of SIGGRAPH '98: Conference Abstracts and Applications. Boston, Massachusetts. ACM Press, 117.

7. Krueger, W., and Froelich, B., 1994. The responsive workbench. IEEE Computer Graphics and Applications, 14(3), 12-15.

8. Latta, J.N., and Oberg, D.J., 1994. A conceptual virtual reality model. IEEE Computer Graphics and Applications, 23-29.

9. Lombard, M., and Ditton, T., 1997. At the heart of it all: The concept of presence. Journal of ComputerMediated Communication [Online], 3(2), Available from

http://www.ascusc.org/jcmc/vol3/issue2/lombard.html [Accessed 17 May 2000].

10. Raskar, R., Welch, G., and Chen, W.-C., 1999. Table-top spatially-augmented reality: Bringing physical models to life with projected imagery. In: Proceedings of International Workshop on Augmented Reality IWAR'99.

11.Slater, M., and Wilbur, S., 1997. A framework for immersive virtual environments (FIVE): Speculations on the role of presence in virtual environments. Presence Teleoperators and Virtual Environments, 6(6), 603616.

12.Sutherland, I.E., 1968. A head mounted three dimensional display. In: Proceedings of the fall joint computer conference (AFIPS). 33(1), 757-764.

13.Welch, R.B., and Warren, D.H., 1980. Immediate perceptual response to intersensory discrepancy. Psychological Bulletin, 88(3), 638-667.

14.Witmer, B.G., and Singer, M.J., 1998. Measuring presence in virtual environments: A presence questionnaire. Presence Teleoperators and Virtual Environments, 7(3), 225-240. 